\title{
Contractile Force of Human Extraocular Muscle: A Theoretical Analysis
}

\author{
Hongmei Guo, Zhipeng Gao, and Weiyi Chen \\ College of Mechanics, Shanxi Key Laboratory of Material Strength \& Structural Impact, Institute of Applied Mechanics \\ and Biomedical Engineering, Taiyuan University of Technology, Taiyuan, Shanxi 030024, China
}

Correspondence should be addressed to Weiyi Chen; chenweiyi211@163.com

Received 11 December 2015; Accepted 2 March 2016

Academic Editor: Yongping Zheng

Copyright (C) 2016 Hongmei Guo et al. This is an open access article distributed under the Creative Commons Attribution License, which permits unrestricted use, distribution, and reproduction in any medium, provided the original work is properly cited.

Aim. The length-contractile force relationships of six human extraocular muscles (EOMs) in primary innervations should be determined during eye movement modeling and surgery of clinical EOMs. This study aims to investigate these relationships. Method. The proposal is based on the assumption that six EOMs have similar constitutive relationships, with the eye suspended in the primary position. The constitutive relationships of EOMs are obtained by optimizing from previous experimental data and the theory of mechanical equilibrium using traditional model. Further, simulate the existing experiment of resistance force, and then compare the simulated results with the existing experimental results. Finally, the mechanical constitutive relationships of EOMs are obtained. Results. The results show that the simulated resistance forces from the other four EOMs except for the horizontal recti well agree with previous experimental results. Conclusion. The mechanical constitutive relationships of six EOMs in primary innervations are obtained, and the rationality of the constitutive relationships is verified. Whereafter, the active stressstrain relationships of the six EOMs in the primary innervations are obtained. The research results can improve the eye movement model to predict the surgical amounts of EOMs before EOM surgery more precisely.

\section{Introduction}

Eye movement is controlled by the six extraocular muscles (EOMs), namely, lateral rectus (LR), medial rectus (MR), superior rectus (SR), inferior rectus (IR), superior oblique (SO), and inferior oblique (IO). This biological regulation mainly depends on the effect of the active forces of the EOMs, which are governed by innervations. Such information helps in understanding the pathology of some eye diseases, for example, strabismus [1]. However, how innervations govern the activation of EOMs is unknown [2-4]. During eye movement modeling and ophthalmic surgery, the primary position (looking straight ahead) [5] as the reference position is important. According to Hering's law, when one of the two eyes fixes on the primary position actively, the innervations of the EOMs of the other eye, which is rotated to any position passively by an external force, are the same as the innervations of the corresponding EOMs of the active eye. In this case, the innervations of EOMs of the active eye and the passive eye are both called primary innervations. The length-contractile force relationships of the six human EOMs in primary innervations are essential for eye movement modeling and EOM surgery. However, the active forces of the EOMs are hardly measured by the experimental installation because of the difficulty in anatomy $[6,7]$. A mathematical model is often used to determine the mechanical properties of biological tissues and biomaterials [8-11]. The present work focuses on calculating the length-contractile force relationships of the EOMs in the primary innervations based on the mechanical equilibrium and the mathematical optimization.

Biomechanical tests of the horizontal EOMs (i.e., LR and MR muscles) have been performed by previous investigators [12-16]. Collins et al. [12,14] determined the length-tension relationships of LR and MR muscles during strabismus surgery, respectively. The experimental surgeries include the horizontal recti of the experimental eye detached from the eyeball and connected by a transducer to measure its force with the normal eye fixed on the given target. Collins et al. [13] also determined the length-tension relationships of the horizontal recti of the normal human subject using a noninvasive 


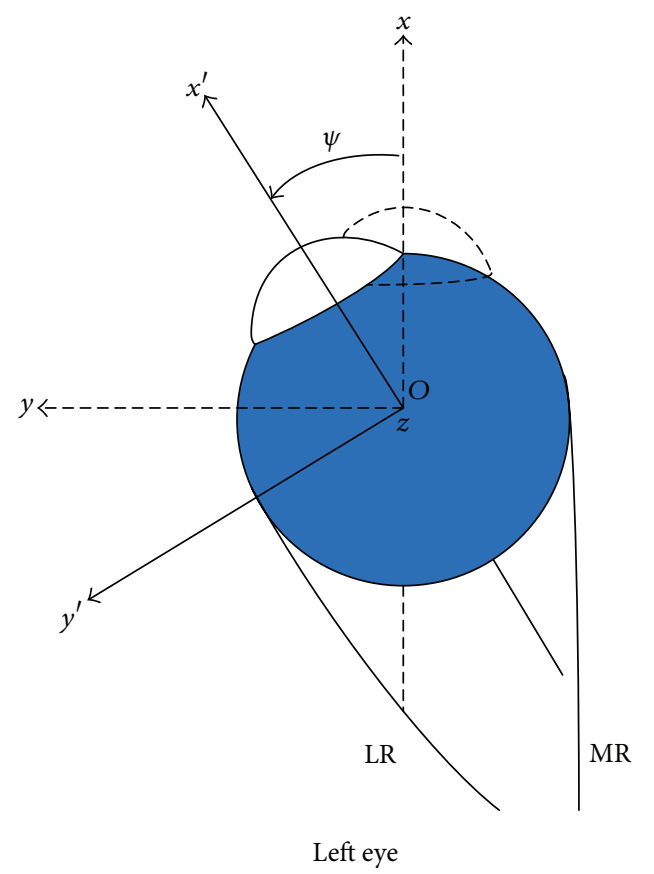

FIGURE 1: Schematic of horizontal movement of the left eye, in which only the lateral rectus (LR) and the medial rectus (MR) are plotted. $x(x, y, z)$ is the reference configuration, and $x^{\prime}\left(x^{\prime}, y^{\prime}, z\right)$ is the current configuration after the eye rotating by $\psi$.

method. Recently, Lennerstrand et al. [15] determined the isometric forces of the human horizontal recti attached onto or detached from the eyeball. They reported that the values of these two forces have no consistent statistical differences. The same group investigated the isometric forces of the EOMs of the saccadic eye and found almost identical forces developed by tendons and pulleys [16].

In recent years, numerous eye movement models have been presented. A mechanical model proposed by Pascolo and Carniel [5] uses a modified Hill muscle model to simulate EOMs. The famous software of eye movement is Orbit ${ }^{\mathrm{TM}}$ 1.8 Gaze Mechanics Simulation, in which the EOMs model is established mainly by referring to the work of Robinson [17] and the work of Miller and Robinson [18, 19]. In the EOMs model of Orbit 1.8, the length-active force relationship and the length-passive force relationship of the LR are fitted by experimental data, and the corresponding relationships of the other EOMs are obtained by multiplying the relative relationships of LR by scale factors [20]. Wei et al. [2] further develop a novel eye movement model, in which the lengthtension relationships of the SR, IR, SO, and IO are obtained based on the length-tension relationships of LR (based on Orbit 1.8) and scaled by their cross sections. In existing eye movement model, only the length-tension relationships of horizontal recti are obtained from experiment, while the length-tension relationships of the other EOMs are still at the exploration stage because few experiment data exist. But modeling in the eye movement requires knowledge of the mechanical properties of all six EOMs.

To improve the set of the mechanical constitutive relationships of the EOM model in eye movement software (e.g., Orbit 1.8) and provide a theoretical direction to the operational decisions of strabismus surgery, the present study focuses on the length-contractile force relationships of the EOMs in the primary innervations, specifically the relationships of the other four EOMs, except for horizontal recti.

\section{Methods}

The contractile forces are calculated based on an optimization proposed herein and on the theory of mechanical equilibrium using the traditional model of eye movement. The geometry model and the coordinate system are shown in Figure 1, in which the geometry parameters (i.e., origins, insertions, and cross sections) of EOMs are the same as those in the work of Gao et al. [21].

2.1. Translation Relationships of the Key Points of EOMs. During the horizontal eye movement, the origins and the insertions of the EOMs can be translated from their reference configurations to current configurations by (1). Additionally, coordinates of origins are the same between two different configurations in the coordinate system

$$
\left(\begin{array}{l}
x \\
y \\
z
\end{array}\right)=\left[\begin{array}{ccc}
\cos \psi & -\sin \psi & 0 \\
\sin \psi & \cos \psi & 0 \\
0 & 0 & 1
\end{array}\right]\left(\begin{array}{l}
x^{\prime} \\
y^{\prime} \\
z
\end{array}\right) .
$$

The tangent points of the EOMs can be calculated using

$$
\begin{aligned}
& x_{C_{i}}^{2}+y_{C_{i}}^{2}+z_{C_{i}}^{2}=R^{2}, \\
& x_{C_{i}} \cdot x_{D_{i}}+y_{C_{i}} \cdot y_{D_{i}}+z_{C_{i}} \cdot z_{D_{i}}=R^{2},
\end{aligned}
$$




$$
\begin{aligned}
& \left(y_{D_{i}} z_{B_{i}}-z_{D_{i}} y_{B_{i}}\right) \cdot x_{C_{i}}+\left(z_{D_{i}} x_{C_{i}}-x_{D_{i}} z_{C_{i}}\right) \cdot y_{C_{i}} \\
& +\left(x_{D_{i}} y_{C_{i}}-y_{D_{i}} x_{C_{i}}\right) \cdot z_{C_{i}}=0,
\end{aligned}
$$

where the subscripts $B_{i}, C_{i}$, and $D_{i}$ represent insertion, tangent point, and origin of the $i$ th EOM, respectively. The in-order Arabic numerals 1-6 are introduced to represent the LR, MR, SR, IR, SO, and IO muscle, respectively.

2.2. Total Force. By fitting the experimental data of the work of Collins [12], the total force of the LR muscle $\left(F_{1}\right)$ in the primary innervation can be described as

$$
F_{1}=5.20 \Delta L_{1}+10.66 e^{\Delta L_{1} /(-6.32)}+8.49
$$

where $\Delta L_{1}$ denotes the elongation of the LR muscle relative to its primary length $L_{11}$, which is $50.21 \mathrm{~mm}$.

The corresponding stress-strain relationship of the LR muscle is

$$
\sigma_{1}=15.61 \varepsilon_{1}+0.64 e^{-7.94 \varepsilon_{1}}+0.51,
$$

where $\sigma_{1}=F_{1} / A_{1}$ is the total stress of the LR muscle, $A_{1}$ $=16.73 \mathrm{~mm}^{2}$ is the cross section of the LR muscle [5], and $\varepsilon_{1}=\Delta L_{1} / L_{11}$ is the strain along the length direction of the LR.

By assuming that the other EOMs have similar stressstrain relationships to that of the LR muscle in the primary innervation, the stress of the $i$ th $\operatorname{EOM}(i=2,3,4,5,6)$ can be described as

$$
\sigma_{i}=\sigma_{i 0}+d \sigma_{i}
$$

where $\sigma_{i 0}=15.61 \varepsilon_{i}+0.64 \exp \left(-7.94 \varepsilon_{i}\right)+0.51$ and $d \sigma_{i}$ is the supplementary variation.

Therefore, the total force of the $i$ th EOM in the primary innervation is

$$
F_{i}=\sigma_{i} \cdot A_{i},
$$

where $i$ denotes the $i$ th EOM.

In the primary position, the eye is kept at equilibrium with the contributions from the six EOMs. Equations (7a)(7d) are the corresponding valid torque equilibrium equations:

$$
\begin{aligned}
& \sum_{i=1}^{6} m_{x_{i}}=0, \\
& \sum_{i=1}^{6} m_{y_{i}}=0, \\
& \sum_{i=1}^{6} m_{z_{i}}=0,
\end{aligned}
$$

where

$$
\begin{aligned}
& m_{x_{i}}=y_{c_{i}} Z_{i}-z_{c_{i}} Y_{i}, \\
& m_{y_{i}}=z_{c_{i}} X_{i}-x_{c_{i}} Z_{i}, \\
& m_{z_{i}}=x_{c_{i}} Y_{i}-y_{c_{i}} X_{i}, \\
& \left(X_{i}, Y_{i}, Z_{i}\right) \\
& \quad=\left(F_{i} \cdot \frac{x_{D_{i}}-x_{C_{i}}}{\sqrt{*}}, F_{i} \cdot \frac{y_{D_{i}}-y_{C_{i}}}{\sqrt{*}}, F_{i} \cdot \frac{z_{D_{i}}-z_{C_{i}}}{\sqrt{*}}\right), \\
& *=\left(x_{D_{i}}-x_{C_{i}}\right)^{2}+\left(y_{D_{i}}-y_{C_{i}}\right)^{2}+\left(z_{D_{i}}-z_{C_{i}}\right)^{2},
\end{aligned}
$$

where $D_{i}$ and $C_{i}$ are the origin and tangent point of the $i$ th EOM, respectively.

Optimizing function (8) was used to make the value of the stress deviator $d \sigma_{i}$ as small as possible; that is, let the objective function $\Phi$ obtained be its minimum value:

$$
\Phi=\sum_{i=2}^{6}\left(\frac{d \sigma_{i}}{\sigma_{i 0}}\right)^{2}
$$

In the primary position, the values of $d \sigma_{2}-d \sigma_{6}$ in (5) could be obtained using the quadprog function [22] by solving a constrained minimal value of objective function $\Phi$ (with conditions that the total force of each EOM should subject to (7a)-(7d)). And the constitutive relationships (total stress-strain relationships) of the six EOMs in the primary innervations can be rewritten as

$$
\begin{aligned}
& \sigma_{1}=15.61 \varepsilon_{1}+0.64 e^{-7.94 \varepsilon_{1}}+0.51, \\
& \sigma_{2}=15.61 \varepsilon_{2}+0.64 e^{-7.94 \varepsilon_{2}}+0.06, \\
& \sigma_{3}=15.61 \varepsilon_{3}+0.64 e^{-7.94 \varepsilon_{3}}+0.47, \\
& \sigma_{4}=15.61 \varepsilon_{4}+0.64 e^{-7.94 \varepsilon_{4}}+0.31, \\
& \sigma_{5}=15.61 \varepsilon_{5}+0.64 e^{-7.94 \varepsilon_{5}}+0.54, \\
& \sigma_{6}=15.61 \varepsilon_{6}+0.64 e^{-7.94 \varepsilon_{6}}+0.52 .
\end{aligned}
$$

2.3. Validation of Total Force Formula. Because of the difficulty of conducting experiments on human in vivo, only the resistance data of the experiment worked out by Collins [12] can be compared, and the corresponding method is shown in Figure 2. In Figure 2, the left eye, whose horizontal recti are detached, is passively rotated temporalward with an artificial external force $F$, whereas the right eye fixes on the primary position actively. In this paper, the above eye movement model (Figure 1) and the obtained total force formula are used to simulate the experiment of Collins [12]. The forces of the other four EOMs (i.e., SR, IR, SO, and IO) resisting the left eye movement (Figure 2) can be obtained using (6) and (9a)-(9f). Finally, compare the simulated results to the experimental results. 


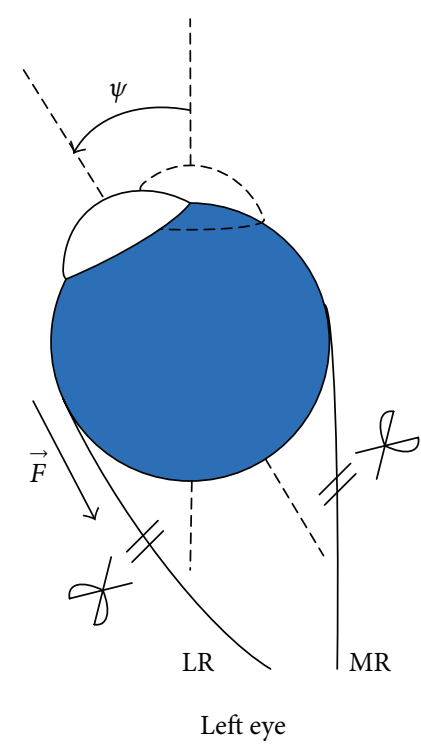

$\vec{F}$ : external force

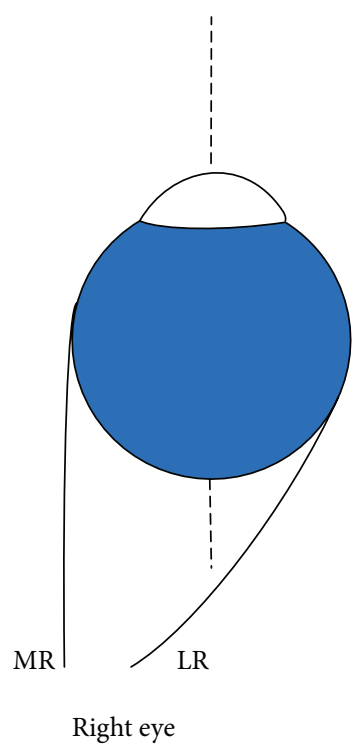

FIGURE 2: Schematic of double eyes only with the lateral rectus (LR) and the medial rectus (MR): not all the six EOMs are plotted.
2.4. Passive Force. By fitting the experimental data of previous works $[6,12,23]$, the passive force $F_{p 1}$ of the LR muscle can be described as

$$
F_{p 1}=-0.29 \Delta L_{1}+4.79 e^{\Delta L_{1} / 4.57}-3.01
$$

The corresponding passive stress $\sigma_{p 1}$ of the LR muscle is

$$
\sigma_{p 1}=-0.87 \varepsilon_{1}+0.29 e^{10.99 \varepsilon_{1}}-0.18 \text {. }
$$

Theoretically, passive stress is only relative to the material, and the passive stresses $\sigma_{p i}$ of all six EOMs can be described as

$$
\sigma_{p i}=-0.87 \varepsilon_{i}+0.29 e^{10.99 \varepsilon_{i}}-0.18
$$

The passive force of the $i$ th EOM is

$$
F_{p i}=\sigma_{p i} \cdot A_{i}
$$

2.5. Active Force. The active stresses $\sigma_{a i}$ of the six EOMs in primary innervations can be obtained by the respective total stress $\sigma_{i}$ (see (9a)-(9f)) minus the passive stress $\sigma_{p i}$ (see (12)); that is,

$$
\begin{aligned}
& \sigma_{a 1}=16.48 \varepsilon_{1}+0.64 e^{-7.94 \varepsilon_{1}}-0.29 e^{10.99 \varepsilon_{1}}+0.69, \\
& \sigma_{a 2}=16.48 \varepsilon_{2}+0.64 e^{-7.94 \varepsilon_{2}}-0.29 e^{10.99 \varepsilon_{2}}+0.24, \\
& \sigma_{a 3}=16.48 \varepsilon_{3}+0.64 e^{-7.94 \varepsilon_{3}}-0.29 e^{10.99 \varepsilon_{3}}+0.65, \\
& \sigma_{a 4}=16.48 \varepsilon_{4}+0.64 e^{-7.94 \varepsilon_{4}}-0.29 e^{10.99 \varepsilon_{4}}+0.49, \\
& \sigma_{a 5}=16.48 \varepsilon_{5}+0.64 e^{-7.94 \varepsilon_{5}}-0.29 e^{10.99 \varepsilon_{5}}+0.72, \\
& \sigma_{a 6}=16.48 \varepsilon_{6}+0.64 e^{-7.94 \varepsilon_{6}}-0.29 e^{10.99 \varepsilon_{6}}+0.70 .
\end{aligned}
$$

The active force of the $i$ th EOM in the primary innervation is

$$
F_{a i}=\sigma_{a i} \cdot A_{i}
$$

\section{Results}

3.1. Total Force. The calculation results shown in Figure 3 reveal that with the eye movement the total force of the MR muscle linearly increases and reaches its maximum value of $617.25 \mathrm{mN}$. Meanwhile, the total force of the LR muscle decreases from $188.74 \mathrm{mN}$ to $45.22 \mathrm{mN}$ with the eye rotating temporalward by $35^{\circ}$ and then slightly increases to $55.23 \mathrm{mN}$, with the eye rotating temporalward by $40^{\circ}$. The total forces of the SR and IR muscles are seemingly stable; that is, their respective minimum values are 123.90 and $147.54 \mathrm{mN}$ in the primary position, and their respective maximum values are 147.93 and $184.72 \mathrm{mN}$ in the temporal $25^{\circ}$. The total forces of the SO and IO muscles are slightly reduced from the primary position to the temporal $40^{\circ}$. Their maximum values are 223.86 and $224.94 \mathrm{mN}$ in the primary position, whereas their minimum values are 154.61 and $127.53 \mathrm{mN}$ in the temporal $40^{\circ}$, respectively.

3.2. Validation Results of Total Force Formula. The comparison results shown in Figure 4 reveal that the calculation results well agree with the experimental data of Collins [12] when the eye rotates temporalward under $25^{\circ}$, which is the normal range of eye abduction [24]. Meanwhile, the errors between the calculation results and experimental data increase with eye rotation from $25^{\circ}$ to $40^{\circ}$.

3.3. The Value of the Objective Function. The values of the objective function $\Phi$ calculated by corresponding formulation (8) are summarized in Table 1 . These values are small 


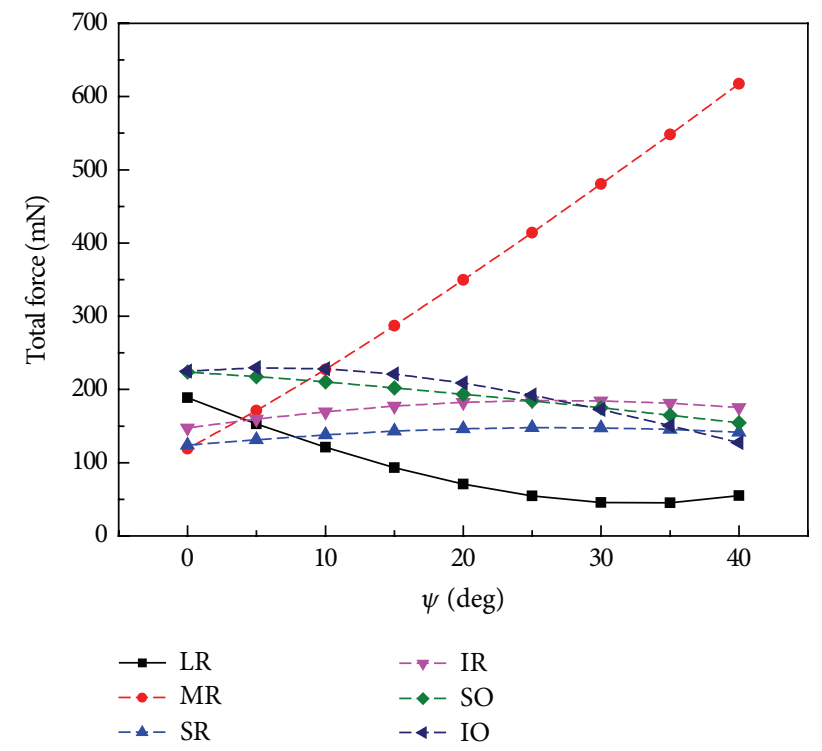

FIGURE 3: Total forces of EOMs with eye passively rotated temporalward in the primary innervations.

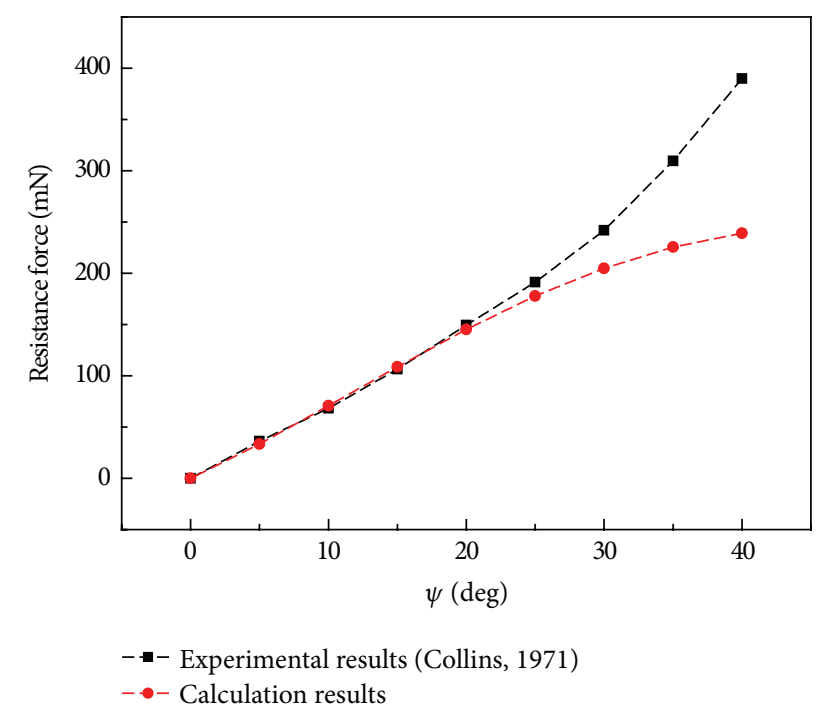

FIGURE 4: Comparison of resistance forces from the other four EOMs, except for the horizontal recti, during passive rotation of the eye in the primary innervations (note: the experimental data are adopted from Figure 27 of reference [12]).

TABLE 1: Values of the objective function in different positions.

\begin{tabular}{lccccccccc}
\hline$\psi(\mathrm{deg})$ & 0 & 5 & 10 & 15 & 20 & 25 & 30 & 35 & 40 \\
\hline$\Phi(-)$ & 0.19 & 0.12 & 0.09 & 0.07 & 0.06 & 0.05 & 0.04 & 0.04 & 0.04
\end{tabular}

enough, and they gradually decrease with the eye temporally rotating from $0^{\circ}$ to $40^{\circ}$.

3.4. Passive Force. Figure 5 shows the calculation results of the passive forces. The passive force of the MR muscles sharply increases from $18.74 \mathrm{mN}$ in the primary position to

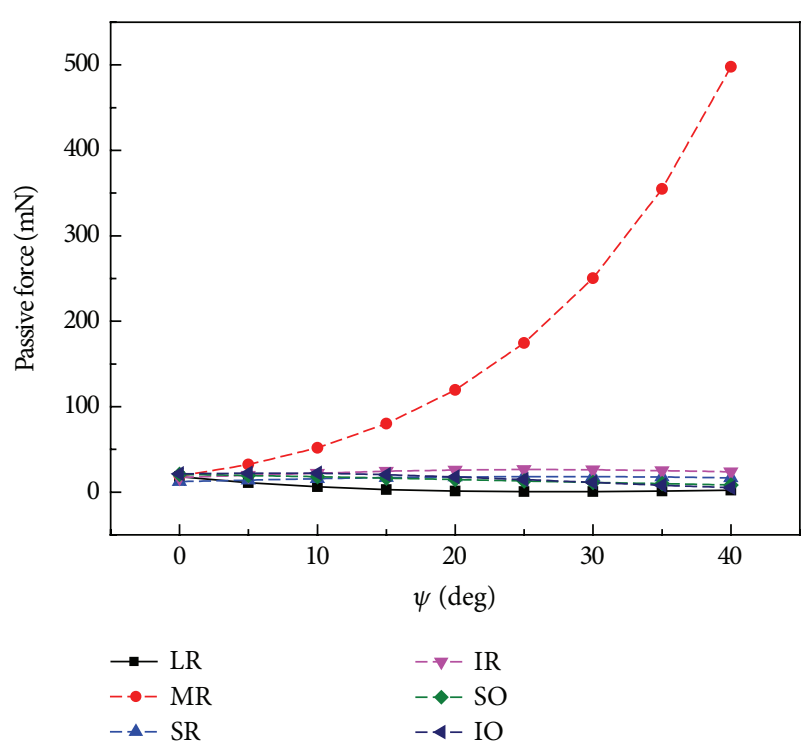

FIGURE 5: Passive forces of EOMs with eye passively rotated temporalward in the primary innervations.

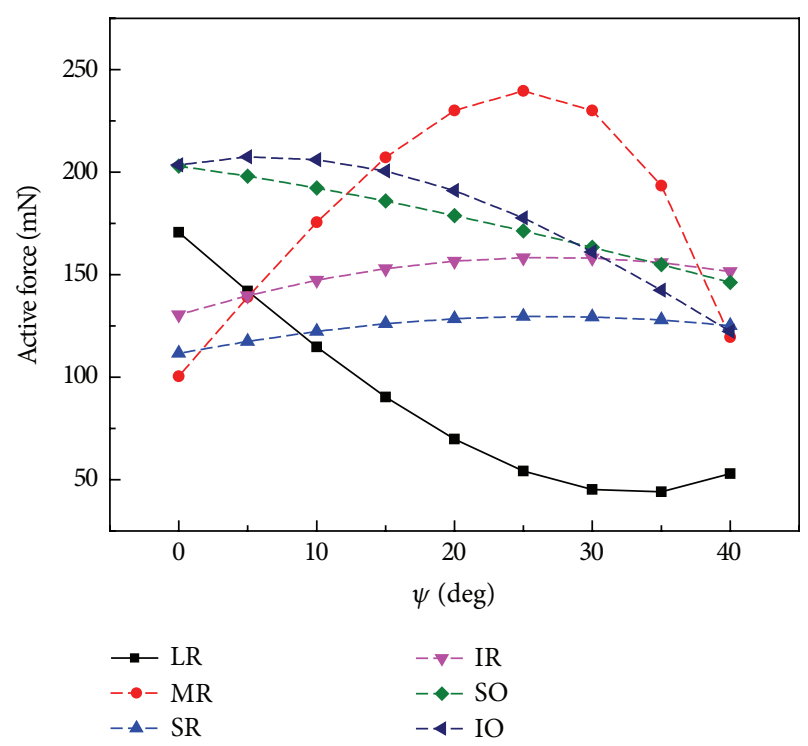

FIgURE 6: Active forces of EOMs with eye passively rotated temporalward in the primary innervations.

$497.66 \mathrm{mN}$ in the temporal $40^{\circ}$, whereas that of the other muscles seems to stabilize within the range of approximately $1-26 \mathrm{mN}$.

3.5. Active Force. The calculation results of the active forces are shown in Figure 6. The active force of the MR muscle increases from $100.45 \mathrm{mN}$ in the primary position to $239.66 \mathrm{mN}$ in the temporal $25^{\circ}$ and then decreases to $119.58 \mathrm{mN}$ in the temporal $40^{\circ}$. By contrast, that of the LR muscle decreases from $170.69 \mathrm{mN}$ in the primary position to $44.15 \mathrm{mN}$ in the temporal $35^{\circ}$ and then rises to $52.97 \mathrm{mN}$ in the temporal $40^{\circ}$. Variations in the vertical recti are very small; that is, the active force of the SR muscle increases 
from $111.64 \mathrm{mN}$ in the primary position to $129.69 \mathrm{mN}$ in the temporal $25^{\circ}$ and then decreases to $125.08 \mathrm{mN}$ in the temporal $40^{\circ}$, whereas that of the IR muscle increases from $130.47 \mathrm{mN}$ in the primary position to $158.33 \mathrm{mN}$ in the temporal $25^{\circ}$ and then decreases to $151.56 \mathrm{mN}$ in the temporal $40^{\circ}$. For the obliques, the active force of the SO muscle decreases from $202.97 \mathrm{mN}$ in the primary position to $146.17 \mathrm{mN}$ in the temporal $40^{\circ}$, whereas that of the IO muscle slightly increases from $203.56 \mathrm{mN}$ in the primary position to $207.38 \mathrm{mN}$ in the temporal $5^{\circ}$ and then decreases to $122.33 \mathrm{mN}$ in the temporal $40^{\circ}$.

\section{Discussion}

In this study, the traditional model is used to simulate the eye movement for simplicity, in which the pulleys of recti are not included. Although many investigators $[25,26]$ have realized the importance of pulleys, the main function of pulleys is that they change the path of the force of recti. The constitutive relationships of EOMs mainly depend on the innervations and the length change of the EOMs. Obviously, pulley plays insignificant role in determining the constitutive relationships of EOMs.

An optimization method is proposed to calculate the actively contractile forces of EOMs in the primary innervations based on the theory of the mechanical equilibrium. The optimizing function is established on the assumption that all six EOMs have similar stress-strain relationships with the eye suspended in primary position. Accordingly, the values of the objective function $\Phi$ should be minimal; these values are shown in Table 1.

The errors between the calculated resistance force and the experimental data apparently and increasingly expand with eye rotation beyond $25^{\circ}$ in Figure 4 . The possible reason is that individual differences among human beings cannot be omitted; that is, the subjects differ in establishing the coordinate of the orbit in this paper $[18,21]$ and performing the previous experiment [12].

Physiologically, the optimal length of a muscle is defined as its length produced at the maximal active force. The optimal length of the skeletal muscle is around its normal resting length [7]. The Hill-type model, a three-element constitutive model of a skeletal muscle, suggests that different innervations lead to varied optimal active forces $[2,27]$. Robinson et al. [28] determined the active forces of the LR muscle. Their measurements revealed that LR has peak active forces when the eye rotated to the nasal by $32^{\circ}$ in different innervations. This result suggests that LR obtains its optimal length with the eye suspended in the nasal by $32^{\circ}$. Interestingly, the present simulation results show that LR has the lowest active force when the eye rotates temporally between $30^{\circ}$ and $35^{\circ}$ (Figure 6). In addition, in the primary innervation with the eye rotating temporally by $25^{\circ}$, the length may be the optimal length of MR because MR achieves its peak value of the active force (Figure 6). The above analysis reveals that the optimal length of the MR is not around its normal resting length, which differs from that of the skeletal muscle. Several investigators have also reported many differences between EOMs and the skeletal muscle, for example, fiber types and biomechanical properties $[6,7,29$, 30].

However, this study cannot determine the optimal lengths of the other four EOMs, whose simulation results reveal fairly ambiguous peak values (Figure 6). To determine the optimal lengths of the SR and IR muscles, eye movement in the vertical plane should be further investigated. The optimal lengths of the SO and IO muscles are also difficult to determine because little is known on simple eye movement as controlled by the oblique.

In the process of clinical strabismus correction, the primary position is crucial. The purpose of strabismus surgery is to make the binocular optical axes parallel and forward in the primary position. The calculation of the surgical amount is also based on the mechanical constitutive relationships of EOMs in the primary position. The abnormal length of some EOMs may lead to strabismus [31-33], and numerous patients suffer from strabismus surgery $[1,34-36]$. The constitutive relationships of the EOMs in primary innervations ((9a)(9f) and (14a)-(14f)) can contribute to the treatment of EOM disorders, such as strabismus. During strabismus surgery, the length and the location of insertion of some EOM may need to be adjusted in the primary innervation, but the operational decisions of the strabismus surgery, such as the adjustment of the length and the location of an EOM, still lack well-defined clinical guidelines [37]. And after the adjustment of the specified EOM, how many forces the other EOMs generate to maintain the eye movement is important. All of the above depend on the mechanical constitutive relationships or length-tension relationships of the six EOMs in primary innervations.

In the existing eye movement models, only the lengthtension relationships of the horizontal recti have been obtained by experiment [12-16]. The length-tension relationships of the other four EOMs were mainly empirical, deduced by that of the horizontal recti $[2,17]$. For example, in the eye movement model proposed by Wei et al. [2], lengthtension relationships of EOMs are obtained from the lengthtension relationships of LR and scaled by their cross sections. The stiffness of the EOMs is occasionally defined as constant $[38,39]$ or the active forces of the EOMs are assumed to be linearly proportional to their innervations [40, 41]. The eye movement model of Pascolo and Carniel [5] modifies Hill's muscle model and skillfully avoids the test difficulty of the real EOM innervations, which can be used to examine healthy case and pathological case such as strabismus based on the dynamical equilibrium. The present simulation is based on the static equilibrium in the primary position. All these examples show that the mechanical constitutive relationships are essential to eye movement modeling. Compared with previous descriptions of the mechanical properties of EOMs, the constitutive relationships of EOMs presented in this study ((9a)-(9f) and (14a)-(14f)) are obtained based on previous experimental data and verified by previous experiments. Evidently, the relationships are closer to reality. The obtained constitutive relationships can be integrated into the eye movement model, which can be used to calculate the surgical amounts of EOMs in strabismus surgery more precisely. 
The ophthalmic diseases, such as the mechanically vertical strabismus [42] and cyclotropia [43] introduced by the congenitally anatomic abnormality, were evaluated qualitatively in the strabismus clinic, but the quantitative analysis will be needed. Therefore, the length-tension relationships of the other EOMs except for the recti are necessary. Because of the difficulty of the human EOMs anatomy and the complicated ethics and morals, the length-tension relationships of the SR, IR, SO, and IO are obviously difficult to measure experimentally. In this paper, the assumption that the six EOMs have the similar stress-strain relationships in the primary position is proposed based on the experiment of strabismus surgery [12]. And the mechanical constitutive relationships of the six EOMs in the primary innervations are deduced. Although the experiment is not conducted and the assumption may be slightly different from the reality, the simulated resistance forces coincide well with the experimental results [12]. Therefore, the reliability of the assumption is verified indirectly.

\section{Conclusion}

To improve eye movement modeling and predict the surgical amount of clinical EOM surgery, this work aimed to determine the mechanical constitutive relationships of six EOMs in primary innervations, particularly the contractile constitutive relationships. Based on previous experimental data of the LR and the assumption that six EOMs have similar strain-stress relationships in primary position, the contractile constitutive relationships of the six EOMs in primary innervations were obtained by combining the equilibrium equations and optimizing functions. The rationality of the proposed assumption and the obtained constitutive relationships were verified through comparisons with previous experimental data. Results indicated that the optimal length of the EOM was not around its normal resting length, which differed from that of the skeletal muscle. Given that the mechanical model of the eye movement system proposed by Pascolo and Carniel would become a routine diagnostic tool of the disturbances of nystagmic movements and strabismus [5], the obtained constitutive relationships of EOMs could be integrated into the eye movement model to predict the surgical amounts of EOMs before EOM surgery.

\section{Competing Interests}

The authors declare that they have no competing interests.

\section{Acknowledgments}

This work was supported by grants from the National Natural Science Foundation of China (nos. 31271005 and 11032008).

\section{References}

[1] M. Y. Chang, F. G. Velez, J. L. Demer, S. J. Isenberg, A. L. Coleman, and S. L. Pineles, "Quality of life in adults with strabismus," American Journal of Ophthalmology, vol. 159, no. 3, pp. 539-544, 2015.
[2] Q. Wei, S. Sueda, and D. K. Pai, "Physically-based modeling and simulation of extraocular muscles," Progress in Biophysics and Molecular Biology, vol. 103, no. 2-3, pp. 273-283, 2010.

[3] R. M. Rashed, S. H. El-Alfy, and I. K. Mohamed, "Histochemical analysis of muscle fiber types of rat superior rectus extraocular muscle," Acta Histochemica, vol. 112, no. 6, pp. 536-545, 2010.

[4] C.-Y. Feng, G. W. Hennig, R. D. Corrigan, T. K. Smith, and C. S. Von Bartheld, "Analysis of spontaneous and nerve-evoked calcium transients in intact extraocular muscles in vitro," Experimental Eye Research, vol. 100, pp. 73-85, 2012.

[5] P. Pascolo and R. Carniel, "From time series analysis to a biomechanical multibody model of the human eye," Chaos, Solitons and Fractals, vol. 40, no. 2, pp. 966-974, 2009.

[6] C. Quaia, H. S. Ying, A. M. Nichols, and L. M. Optican, “The viscoelastic properties of passive eye muscle in primates: I: static and step responses," PLoS ONE, vol. 4, no. 4, Article ID e4850, 2009.

[7] B. J. Kushner, "Incomitant strabismus: does extraocular muscle form denote function?" Archives of Ophthalmology, vol. 128, no. 12, pp. 1604-1609, 2010.

[8] A. E. Ehret, M. Böl, and M. Itskov, "A continuum constitutive model for the active behaviour of skeletal muscle," Journal of the Mechanics and Physics of Solids, vol. 59, no. 3, pp. 625-636, 2011.

[9] A. Kandler and S. Shennan, "A non-equilibrium neutral model for analysing cultural change," Journal of Theoretical Biology, vol. 330, pp. 18-25, 2013.

[10] X. Gao and W. Gu, "A new constitutive model for hydrationdependent mechanical properties in biological soft tissues and hydrogels," Journal of Biomechanics, vol. 47, no. 12, pp. 31963200, 2014.

[11] Z.-L. Zhao, H.-P. Zhao, J.-S. Wang, Z. Zhang, and X.-Q. Feng, "Mechanical properties of carbon nanotube ropes with hierarchical helical structures," Journal of the Mechanics and Physics of Solids, vol. 71, pp. 64-83, 2014.

[12] C. C. Collins, "Orbital mechanics," in The Control of Eye Movements, P. Bach-Y-Rita, C. C. Collins, and J. E. Hyde, Eds., pp. 283-325, Academic Press, New York, NY, USA, 1971.

[13] C. C. Collins, M. R. Carlson, A. B. Scott, and A. Jampolsky, "Extraocular muscle forces in normal human subjects," Investigative Ophthalmology and Visual Science, vol. 20, no. 5, pp. 652-664, 1981.

[14] C. C. Collins, D. O’Meara, and A. B. Scott, "Muscle tension during unrestrained human eye movements," Journal of Physiology, vol. 245, no. 2, pp. 351-369, 1975.

[15] G. Lennerstrand, C. Schiavi, S. Tian, M. Benassi, and E. C. Campos, "Isometric force measured in human horizontal eye muscles attached to or detached from the globe," Graefe's Archive for Clinical and Experimental Ophthalmology, vol. 244, no. 5, pp. 539-544, 2006.

[16] G. Lennerstrand, R. Bolzani, M. Benassi, S. Tian, and C. Schiavi, "Isometric force development in human horizontal eye muscles and pulleys during saccadic eye movements," Acta Ophthalmologica, vol. 87, no. 8, pp. 837-842, 2009.

[17] D. A. Robinson, "A quantitative analysis of extraocular muscle cooperation and squint," Investigative Ophthalmology, vol. 14, no. 11, pp. 801-825, 1975.

[18] J. M. Miller and D. A. Robinson, "A model of the mechanics of binocular alignment," Computers and Biomedical Research, vol. 17, no. 5, pp. 436-470, 1984. 
[19] T. Haslwanter, M. Buchberger, T. Kaltofen, R. Hoerantner, and S. Priglinger, "SEE++: a biomechanical model of the oculomotor plant," Annals of the New York Academy of Sciences, vol. 1039, pp. 9-14, 2005.

[20] J. M. Miller, Orbit ${ }^{\mathrm{TM}} 1.8$ Gaze Mechanics Simulation User's Manual, Eidactics, San Francisco, Calif, USA, 1st edition, 1999, http://www.eidactics.com/.

[21] Z. Gao, H. Guo, and W. Chen, "Initial tension of the human extraocular muscles in the primary eye position," Journal of Theoretical Biology, vol. 353, pp. 78-83, 2014.

[22] Matlab Optimization Toolbox, Tomlab Optimization Inc, San Diego, Calif, USA, http://tomopt.com/.

[23] C. Quaia, H. S. Ying, and L. M. Optican, "The viscoelastic properties of passive eye muscle in primates. III: force elicited by natural elongations," PLoS ONE, vol. 5, no. 3, Article ID e9595, 2010.

[24] P. Pascolo, R. Carniel, and S. Grimaz, "Dynamical models of the human eye and strabismus," Chaos, Solitons and Fractals, vol. 41, no. 5, pp. 2463-2470, 2009.

[25] R. A. Clark, J. M. Miller, and J. L. Demer, “Three-dimensional location of human rectus pulleys by path infections in secondary gaze positions," Investigative Ophthalmology and Visual Science, vol. 41, no. 12, pp. 3787-3797, 2000.

[26] R. A. Clark and J. L. Demer, "Magnetic resonance imaging of the effects of horizontal rectus extraocular muscle surgery on pulley and globe positions and stability," Investigative Ophthalmology and Visual Science, vol. 47, no. 1, pp. 188-194, 2006.

[27] A. V. Hill, "The heat of shortening and the dynamic constants of muscle," Proceedings of the Royal Society B: Biological Sciences, vol. 126, no. 843, pp. 136-195, 1938.

[28] D. A. Robinson, D. M. O'Meara, A. B. Scott, and C. C. Collins, "Mechanical components of human eye movements," Journal of Applied Physiology, vol. 26, no. 5, pp. 548-553, 1969.

[29] L. K. McLoon, J. Rowe, J. Wirtschafter, and K. M. McCormick, "Continuous myofiber remodeling in uninjured extraocular myofibers: myonuclear turnover and evidence for apoptosis," Muscle and Nerve, vol. 29, no. 5, pp. 707-715, 2004.

[30] R. F. Spencer and J. D. Porter, "Biological organization of the extraocular muscles," in Neuroanatomy of the Oculomotor System: Progress in Brain Research, J. A. Büttner-Ennever, Ed., pp. 43-80, Elsevier BV, Amsterdam, Netherlands, 2006.

[31] M. Gräf and B. Lorenz, "Strabismus," Monatsschrift Kinderheilkunde, vol. 163, no. 3, pp. 230-240, 2015.

[32] R. Rabinowitz and J. L. Demer, "Muscle path length in horizontal strabismus," Journal of AAPOS, vol. 18, no. 1, pp. 4-9, 2014.

[33] A. Narasimhan, L. Tychsen, V. Poukens, and J. L. Demer, "Horizontal rectus muscle anatomy in naturally and artificially strabismic monkeys," Investigative Ophthalmology and Visual Science, vol. 48, no. 6, pp. 2576-2588, 2007.

[34] Y. H. Jiao, Y. T. Zhu, Z. Zhou et al., "Strabismus surgery distribution during 10-year period in a tertiary hospital," Chinese Medical Journal, vol. 127, no. 16, pp. 2911-2914, 2014.

[35] H. E. A. Bicas, "A major problem in strabismus and its possible solution," Arquivos Brasileiros de Oftalmologia, vol. 77, no. 4, pp. 250-255, 2014.

[36] J. L. Demer, R. A. Clark, R. Kono, W. Wright, F. Velez, and A. L. Rosenbaum, "A 12-year, prospective study of extraocular muscle imaging in complex strabismus," Journal of AAPOS, vol. 6, no. 6, pp. 337-347, 2002.

[37] L. Gnanaraj and S. R. Richardson, "Interventions for intermittent distance exotropia: review," Eye, vol. 19, no. 6, pp. 617-621, 2005.
[38] S. Schutte, S. P. W. van den Bedem, F. van Keulen, F. C. T. van der Helm, and H. J. Simonsz, "A finite-element analysis model of orbital biomechanics," Vision Research, vol. 46, no. 11, pp. 17241731, 2006.

[39] S. Schutte, J. R. Polling, F. C. T. van der Helm, and H. J. Simonsz, "Human error in strabismus surgery: quantification with a sensitivity analysis," Graefe's Archive for Clinical and Experimental Ophthalmology, vol. 247, no. 3, pp. 399-409, 2009.

[40] C. Quaia and L. M. Optican, "Commutative saccadic generator is sufficient to control a 3-D ocular plant with pulleys," Journal of Neurophysiology, vol. 79, no. 6, pp. 3197-3215, 1998.

[41] T. Raphan, "Modeling control of eye orientation in three dimensions. I. Role of muscle pulleys in determining saccadic trajectory," Journal of Neurophysiology, vol. 79, no. 5, pp. 26532667, 1998.

[42] R. Caputo, F. Tinelli, A. Bancale et al., "Motor coordination in children with congenital strabismus: effects of late surgery," European Journal of Paediatric Neurology, vol. 11, no. 5, pp. 285291, 2007.

[43] J. Felius, K. G. Locke, M. A. Hussein, D. R. Stager Jr., and D. R. Stager Sr., "Photographic assessment of changes in torsional strabismus," Journal of AAPOS, vol. 13, no. 6, pp. 593-595, 2009. 


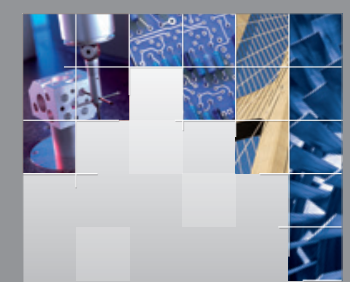

\section{Enfincering}
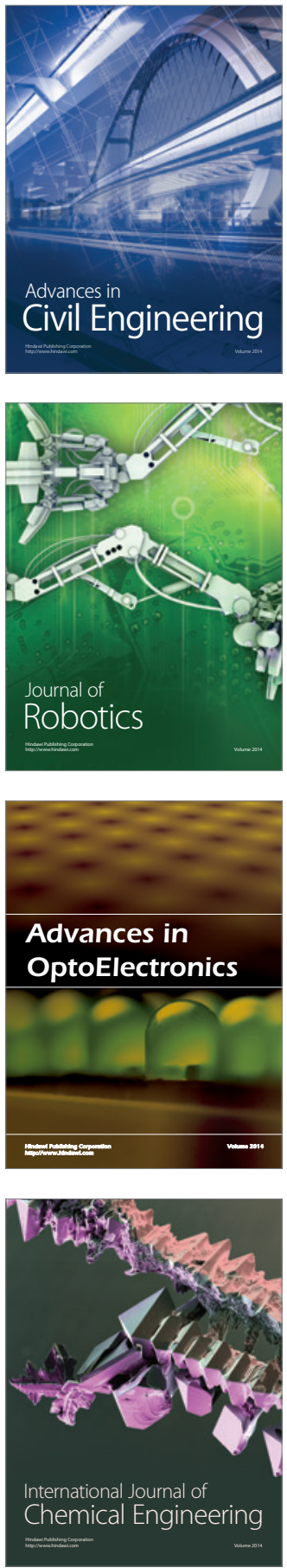

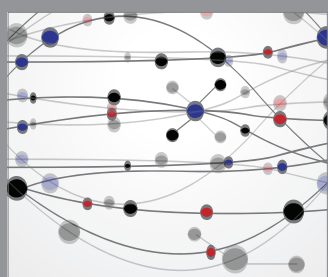

The Scientific World Journal

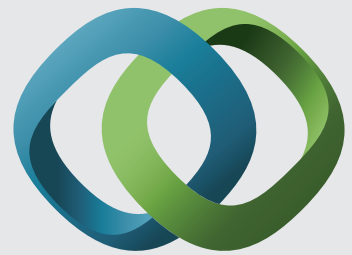

\section{Hindawi}

Submit your manuscripts at

http://www.hindawi.com
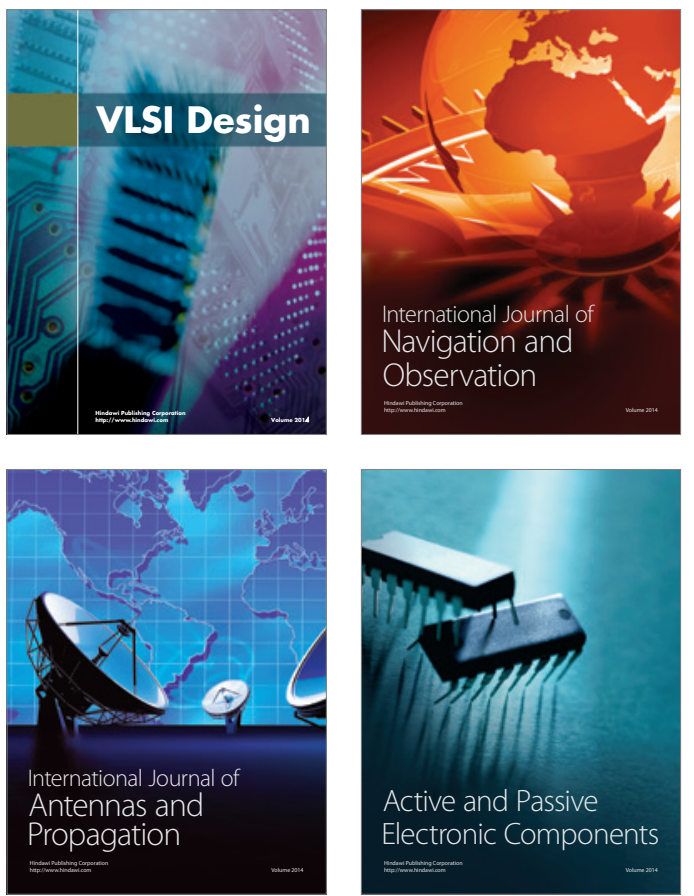


International Journal of

Distributed

Sensor Networks

Journal of

Control Science

and Engineering
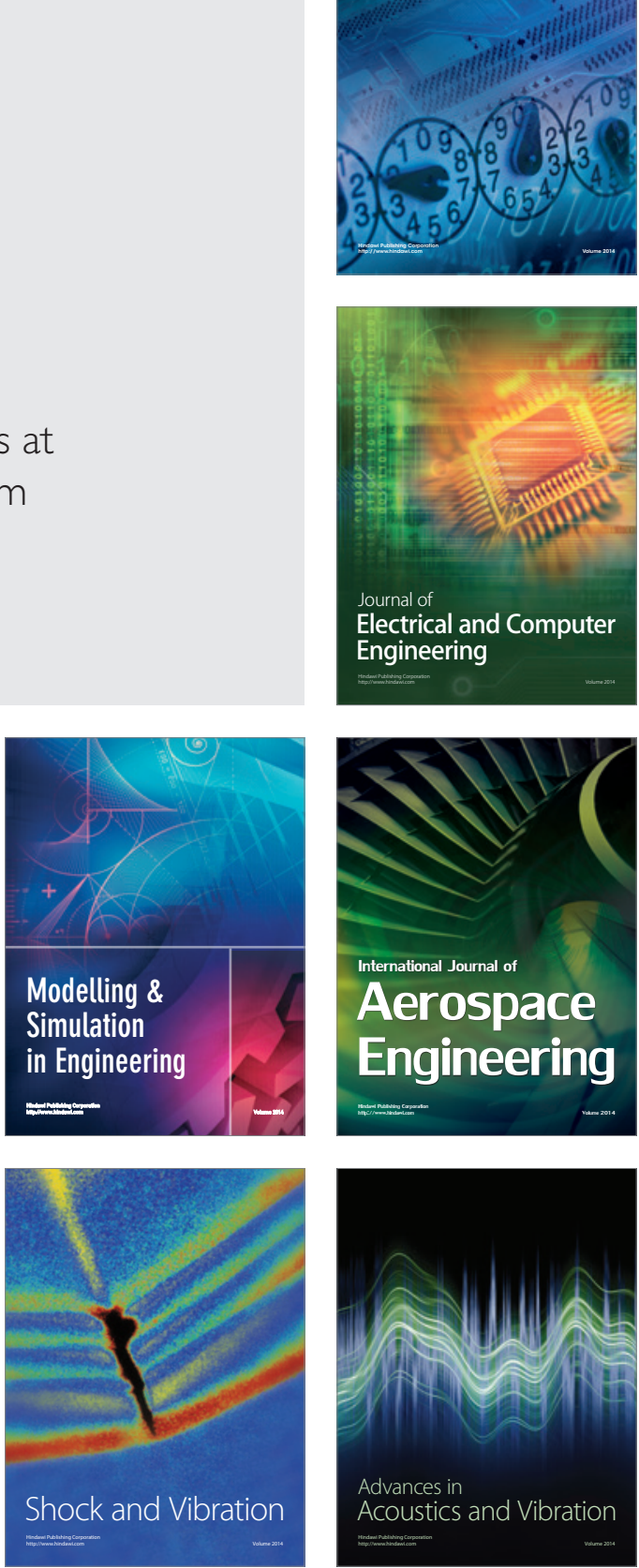